\title{
Robust Identification using Decimation with Application to Large Space Structure
}

\author{
Shuichi Adachi and Yoshihiro Sunaga \\ Faculty of Engineering, Utsunomiya University \\ 2753 Ishii, Utsunomiya, 321 Japan \\ e-mail adachi@jsrv01.utsunomiya-u.ac.jp
}

\begin{abstract}
In the control system design, the robust control theory has been studied actively. However, in order to apply the robust control theory to real plants, it is necessary to evaluate model uncertainty as well as a nominal model. In this paper, a new and practical identification method for the model uncertainty is proposed. The identification method is based on the ordinary leastsquares identification method in cooperation with the decimation. The key idea of the method is a frequency division by the decimation. The frequency band of the plant is divided into two parts, that is, one corresponds to the nominal model and the other corresponds to unmodeled dynamics. Effectiveness of the method is examined through actual data that is collected thorogh identification experiments of a ground-based test model for a large space structure (LSS).
\end{abstract}

\section{INTRODUCTION}

In the control system design, the robust control theory, especially the $H_{\infty}$ control, has been studied actively and many application examples have been reported in various $\operatorname{areas}^{[1]}$. However, in order to apply the robust control theory to real plants, it is necessary to evaluate model uncertainty as well as a nominal model, so the researches for the robust identification, which means system identification for the robust control system design, have become one of the hottest topics in the system identification ${ }^{[2]}$.

In this paper, the robust identification for a large space structure (LSS) is considered. The importance of on-orbit identification of space structures has been widely recognized according to the enlargement of them ${ }^{[3]}$. Because the on-orbit characteristics of an LSS are not sufficiently identified by either the ground-testing or computer generated modeling techniques, e.g., the finite element method (FEM). Therefore, the approach based on the system identification is considered to become one of the most promsing on-orbit identification method.

The LSS is distributed parameter system in nature, so infinity modes exist. However, the control system, e.g., an attitude control system, is designed based on the reducedorder model. After all, higher modes are neglected in the nominal model, and it is considered that they cause the model uncertainty. In this case, it is natural to use the additive uncertainty description. So, the purpose of this paper is to propose a new robust identification method which evaluates the frequency characteristics of the additive uncertainty.

Main advantage of the proposed robust identification method is that it uses no a priori information on the uncertainty such that the upper bound of the model uncertainty. The key idea of the method is a frequency division of the plant, that is, the LSS, by the decimation. It is well known that the decimation is one of the most important tools in the multirate signal processing, and it is also useful tool in the system identification $^{[4]}$. By using the decimation, the whole frequency band of the plant is divided into two parts, one corresponds to the nominal model in the low frequency band and the other corresponds to the additive uncertainty in the high frequency band. Then, in order to estimate frequency responses, the ordinary least-squares method ${ }^{[5]}$ in cooperation with suitable regularization is applied to both the decimated input-output data and the original one. Finally, frequency characteristics of the additive uncertainty can be evaluated based on the two identified frequency responses.

In order to show effectiveness of the proposed method, 
it is applied to identification experimental data by a ground-based test model for the LSS. The experimental results indicate that the proposed robust identification method is easy to use, and accurate evaluation for the model uncertainty is accomplished.

\section{PROBLEM FORMULATION}

\subsection{Additive Uncertainty Representation}

The transfer function, say $P(s)$, from the input torque to the attitude angle of the LSS is given by

$$
P(s)=\frac{1}{I s^{2}}+\sum_{i=1}^{\infty} \frac{\phi_{i}^{2}}{s^{2}+2 \zeta_{i} \omega_{i} s+\omega_{i}^{2}}
$$

where $I$ is the inertia moment of a rigid body, $\left\{\omega_{i}\right\}$ is the natural frequency of the elastic mode, $\left\{\zeta_{i}\right\}$ is the damping ratio and $\left\{\phi_{i}\right\}$ is the torque admittance ${ }^{[\theta]}$. Since the LSS is distributed parameter system in nature, the transfer function Eq. (1) has infinity elastic modes. In the control system design point of view, however, the controller is designed based on the main lower elastic modes, so the infinity elastic modes are truncated by the reduced-order transfer function. It is well known that the higher mode truncation may cause the spillover instability, especially the accurate control performance, i.e., higher control band width, is specified ${ }^{[7]}$. Therefore, the robust control design has been usually applied to the LSS in recent years.

Although there are a lot of representations for the model uncertainty, e.g., the multiplicative one, the additive one, and so on, it is natural for the LSS given by Eq. (1) to use the following additive uncertainty representation.

$$
P(s)=P_{\text {nom }}(s)+\Delta_{A}(s) W_{A}(s)
$$

where $P_{\text {nom }}(s)$ is the nominal model and $\Delta_{A}(s) W_{A}(s)$ is the additive uncertainty, given by

$$
\begin{aligned}
& P_{\text {nom }}(s)=\frac{1}{I s^{2}}+\sum_{i=1}^{m} \frac{\phi_{i}^{2}}{s^{2}+2 \zeta_{i} \omega_{i} s+\omega_{i}^{2}} \\
& \Delta_{A}(s) W_{A}(s)=\sum_{i=m+1}^{\infty} \frac{\phi_{i}^{2}}{s^{2}+2 \zeta_{i} \omega_{i} s+\omega_{i}^{2}}
\end{aligned}
$$

respectively. $\Delta_{A}(s)$ is a stable transfer function which satisfies $\left\|\Delta_{A}(s)\right\|_{\infty} \leq 1$ and $W_{A}(s)$ is a stable frequency weighting function. The block diagram of the Eq. (2) is shown in Fig.1, where $d_{s}(t)$ denotes the stochastic disturbance. For example, in the deterministic robust identification, $d_{d}(t)$, i.e., the output from the additive uncertainty $\Delta_{A}(s) W_{A}(s)$, is assumed bounded deterministic disturbance.

The robust identification problem considered in this paper is how to identify the nominal model as well as the frequency weighting function which specifies the model uncertainty, based on finite input-output data.

\subsection{Discrete-Time Identification Model}

In order to apply the least-squares identification method, the continuous-time system given by Eq. (1) is transformed into a discrete-time model

$$
A(q) y(k)=B(q) u(k)+e(k)
$$

by using a sampler with sampling time $T$ and a zero-order holder, where $u(k)$ is the input, $y(k)$ is the output, and $e(k)$ is the disturbance which is considered unknown but bounded disturbance. The polynomial $A(q)$ and $B(q)$ are given by

$$
A(q)=1+a_{1} q^{-1}+\cdots+a_{n} q^{-n}, \quad B(q)=b_{1} q^{-1}+\cdots+b_{n} q^{-n}
$$
respectively, where $q^{-1}$ is the backward shift operator, i.e. $q^{-1} u(k)=u(k-1)$.

Now, Eq.(4) can be rewritten as the following linear regression form.

$$
y(k)=\theta^{T} x(k)+e(k)
$$

where $\theta=\left[a_{1}, \ldots, a_{n}, b_{1}, \ldots, b_{n}\right]^{T}$

$$
x(k)=[-y(k-1), \ldots,-y(k-n), u(k-1), \ldots, u(k-n)]^{T}
$$

and $\theta$ is the unknown parameter vector to be estimated. In this paper, $\theta$ is estimated by using the ordinary leastsquares method. Once $\theta$ is estimated, the pulse transfer function estimate is constructed by

$$
\hat{G}(q)=\frac{\hat{B}(q)}{\hat{A}(q)}
$$

Moreover, the frequency response estimate is calculated as

$$
\hat{G}(j \omega)=\left.\frac{\hat{B}(q)}{\hat{A}(q)}\right|_{q=\exp (-j \omega T)}
$$

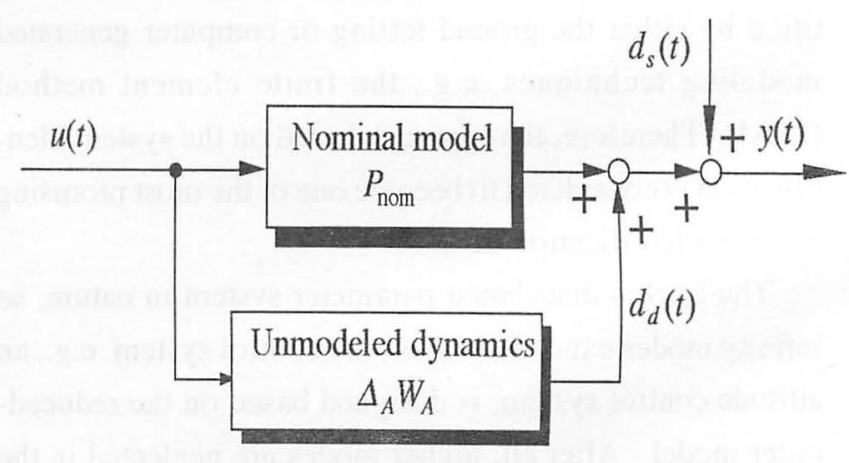

Fig.1 Additive uncertainty representation 


\section{IDENTIFICATION OF MODEL UNCERTAINTY BY USING DECIMATION}

In the robust identification method, it is frequently assumed that a priori information on the model uncertainty is available. For example, Kosut, et al. assumed that a frequency weighting function which specifies the multiplicative uncertainty is given a priori so as to evaluate the parameter set for the nominal model ${ }^{[2]}$. However, such a priori information is unrealistic in many application cases, because the robust control design can be applied if the multiplicative frequency weighting function is available.

In this paper, we propose a new and practical robust identification method which uses no a priori information. The key idea of the method is a frequency division of the plant by the decimation which is one of the most important signal processing techniques as well as the interpolation. The sampling period $T$ is transformed to $d T$ by the decimation, where $d$ is an integer such that $d \geq 2$. By using the decimation, the whole frequency band of the plant is divided into two parts, one corresponds to the nominal model in the low frequency band and the other corresponds to the additive uncertainty in the high frequency band. The frequency characteristics for the additive uncertainty is then evaluated from frequency response estimates based on the original data minus the one based on the decimated data.

\section{A new robust identification method}

The procedure for a new robust identification method using the decimation is summarized as follows.

Step 1: System identification by using high order model (i) Collect input-output data $\{u(k), y(k) ; k=1,2, \ldots, N\}$ of the plant using a sampler with sampling period $T$.

(ii) Fit the data for a high order model based on the ordinary least-squares method. If the data is contaminated by noise, it is better to use a very high order model in combination with suitable regularization, since it alleviates the numerical ill-conditioning ${ }^{[8]}$.

Now, we consider the cost performance

$$
J(N)=\frac{1}{N} \sum_{k=1}^{N}\left\{y(k)-x^{T}(k) \hat{\theta}\right\}^{2}+\rho(N) \|\left.\hat{\theta}\right|^{2}
$$

where $\rho(N)$ is a nonnegative constant which is called the regularization constant. Note that Eq. (8) is closely related to the Ridge estimation in the statistical theory. Then, the constrained least-squares estimates $\hat{\theta}$ that minimizes Eq. (8) is given by the solution of the well-known normal equation

where

$$
\hat{\theta}(N)=\boldsymbol{R}^{-1}(N) \boldsymbol{g}(N)
$$

$$
\boldsymbol{R}(N):=\boldsymbol{F}(N)+\rho(N) \boldsymbol{I}=\frac{1}{N} \sum_{k=1}^{N} \boldsymbol{x}(k) \boldsymbol{x}^{T}(k)+\rho(N) \boldsymbol{I}
$$

Thus, $\rho(N)$ is added into the diagonal elements of the covariance matrix $F(N)$ to mitigate the non-singularity of $F(N)$. Actually, however, $\rho(N)$ is usually chosen empirically. The optimal choice of the regularization constant is ommited ( see [8]). The recursive least-squares method with the regularization is given as follows.

$$
\begin{aligned}
& \hat{\theta}(k)=\hat{\theta}(k-1)+\boldsymbol{P}(k) \boldsymbol{x}(k)\left\{y(k)-\boldsymbol{x}^{T}(k) \hat{\theta}(k-1)\right\} \\
& \boldsymbol{P}(k)=\boldsymbol{P}(k-1)-\frac{\boldsymbol{P}(k-1) \boldsymbol{x}(k) \boldsymbol{x}^{T}(k) \boldsymbol{P}(k-1)}{1+\boldsymbol{x}^{T}(k) \boldsymbol{P}(k-1) \boldsymbol{x}(k)}
\end{aligned}
$$

where $\boldsymbol{P}(0)=\left(1 / \rho_{0}^{*}\right) \boldsymbol{I}$ and $\rho_{0}^{*}$ is a regularization constant at time 0. Note that the recursive algorithm, Eq.(11) coincides with the ordinary least-squares algorithm. The only difference is the initial value of the covariance matrix.

(iii) Based on the estimated parameters, the frequency response estimate is calculated by Eq. (7), that is,

$$
\hat{G}_{\text {true }}(j \omega)=\left.\frac{\hat{B}(q)}{\hat{A}(q)}\right|_{q=\exp (-j \omega T)}
$$

In this paper, Eq. (12) is assumed to express a frequency response estimate of the true plant.

Step 2: Identification of nominal model by decimation

(i) Decimate the input-output data collected in Step 1. The new sampling period $T^{\prime}$ is determined in considering a frequency band in which the nominal model dynamics of the plant is included. It is important that the detemination of $T^{\prime}$ is not an identification point of view but a control design point of view. Note that the effect of the additive model uncertainty which exists in a high frequency band, e.g., residual modes of the LSS, is removed by the decimation. So, the decimation plays a frequency division role.

(ii) Apply the least-squares method to the decimated inputoutput data in order to estimate parameters, say $\theta$, for a discrete-time model with a sampling period $T^{\prime}$. Based on the estimates $\hat{\theta}^{\prime}$, the estimates of polynomials are con- 
structed as follows.

$$
\begin{aligned}
& \hat{A}^{\prime}(q)=1+\hat{a}_{1}^{\prime} q^{-1}+\cdots+\hat{a}_{n}^{\prime} q^{-n} \\
& \hat{B}^{\prime}(q)=\hat{b}_{1}^{\prime} q^{-1}+\cdots+\hat{b}_{n}^{\prime} q^{-n}
\end{aligned}
$$

(iii) Based on the identified model polynomials, Eq. (13), modal parameters of the nominal model can be calculated [6]

(iv) According to the modal parameters calculated above, calculate a discrete-time model with a sampling period $T$, say $\hat{G}_{\text {nom }}(q)$.

\section{Step 3:Evaluation of the nomparametric uncertainty}

Based on the results of Step 1 and 2, two nonparametric model uncertainties are obtained as follows.

\section{(i) Additive uncertainty}

According to Eq. (2),

$$
\Delta_{A}\left(e^{-j \omega T}\right) W_{A}\left(e^{-j \omega T}\right)=\hat{G}_{\text {true }}\left(e^{-j \omega T}\right)-\hat{G}_{\text {nom }}\left(e^{-j \omega T}\right)
$$

is derived. Then, determine a weighting function for the additive uncertainty $W_{A}\left(e^{-j \omega T}\right)$ which covers $\left|\Delta_{A}\left(e^{-j \omega T}\right) W_{A}\left(e^{-j \omega T}\right)\right|$ in the frequency domain.

\section{(ii) Multiplicative uncertainty}

The multiplicative uncertainty

$$
\Delta_{M}\left(e^{-j \omega T}\right) W_{M}\left(e^{-j \omega T}\right)=\frac{\hat{G}_{\text {true }}\left(e^{-j \omega T}\right)}{\hat{G}_{\text {nom }}\left(e^{-j \omega T}\right)}-1
$$

is derived according to the definition. Then, determine a weighting function $W_{M}\left(e^{-j \omega T}\right)$ for the multiplicative uncertainty which covers $\left|\Delta_{M}\left(e^{-j \omega T}\right) W_{M}\left(e^{-j \omega T}\right)\right|$ in the frequency domain.

The proposed method is based on the fact that the elastic modes for the LSS are arranged from the low frequency to the high one and dominant modes are concentrated in the low frequency band, therefore the nominal model is constructed by the main elastic modes which exist in the lower frequency. The main advantages of the method is that the procedure is so simple that it is easy to apply to practical problems. Also, because the method is based on the leastsquares method in step 1 and 2 , stochastic disturbances due to noise can be eliminated as well as the bias error due to the unmodeled dynamics.

\section{IDENTIFICATION EXPERIMENTS}

In this section, the identification problem of a flexible spacecraft typified by the ETS-VI (Engineering Test Satellite-VI) spacecraft is studied. The ETS-VI, which is illus-

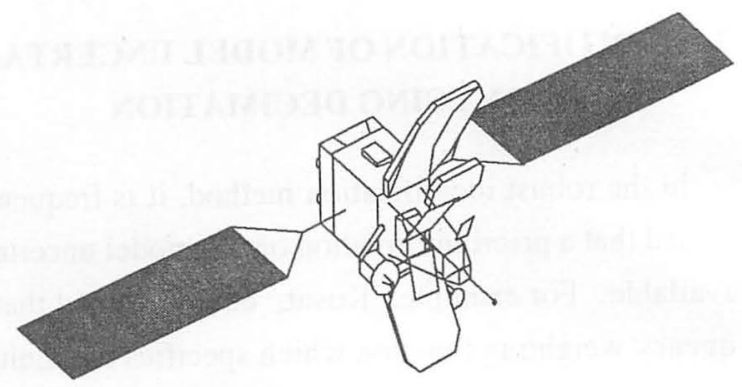

Fig.2 ETS-VI as an example of a large space structure

trated in Fig. 2 is to be launched in August, 1994 in Japan. Because the ETS-VI is a very large space structure, it is impossible to make a ground-based test which simulates the space environment accurately. Therefore, it is necessary to identify the LSS dynamics accurately directly from on-orbit measurements.

\subsection{Laboratory Apparatus}

The ground-based test model for the ETS-VI is shown in Fig.3. It simulated the attitude motion around the roll axis by using a single-axis air table. Two CFRP panels connected by rigid hinges were used as a solar paddle model. These were clamped to the central hub. The rotation angle was measured by a magnetic rotary encoder . The control torque was generated by a DC motor attached to the end of the rotary shaft. The transfer function from the control torque input to the attitude measurement output of the air-table model with four modes (one rigid and three out-of-plane bending modes) obtained by the FEM is dipicted in Fig.4.

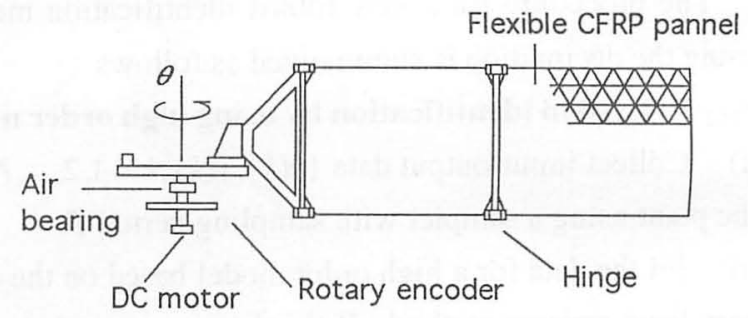

Fig.3 Laboratory apparatus

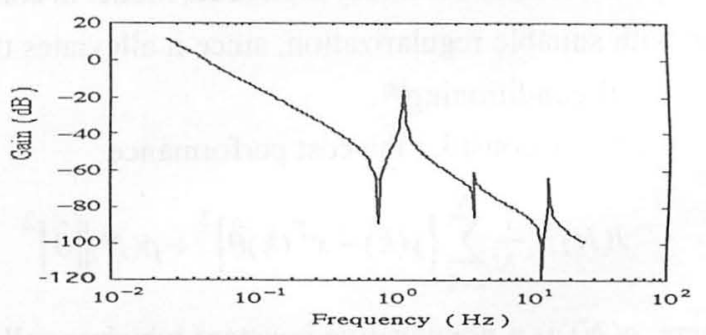

Fig.4 Bode diagram of the experimantal apparatus based on the FEM 

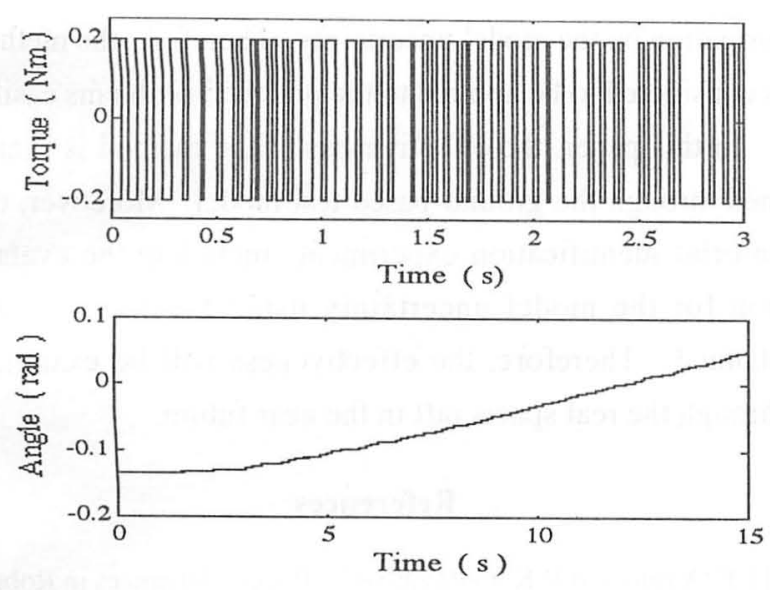

Fig.5 Input-output data obtained by identifation experiment

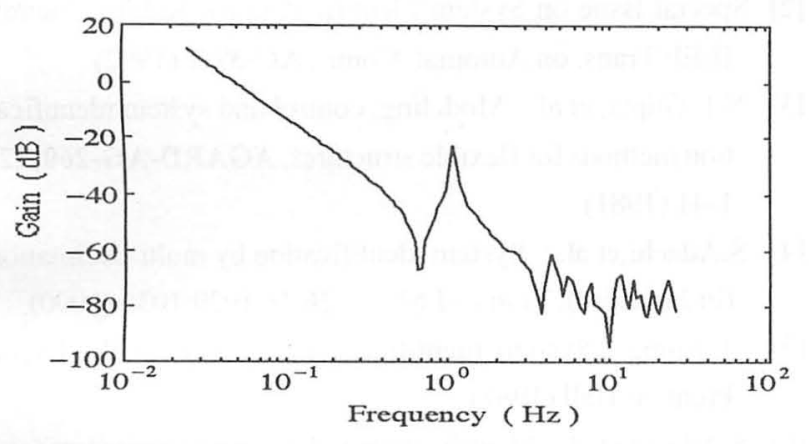

Fig.6 Identified gain response of the true plant by using 50 th order model

\subsection{Identification Results for Model Uncertainty}

The sampling time $T$ was set to $0.02(\mathrm{sec})$. The $\mathrm{M}$ sequence, which is well-known pseudo-random binary signal(PRBS), was used as the input torque sequence for identification experiments. The input and output signals collected in an identification experiment are shown in Fig.5. Because the rigid body dynamics, which correspond to double poles at $s=0$ in the s-plane, are dominant, measurements of the output angle are divergent. So, the duration time of the identification experiment is constrained, in this case the number of data is only 750 . Then the proposed robust identification method is applied to the input-output data in Fig.5.

Step 1 : Frequency response is estimated by the leastsquares method with 50 th order model. The identified gain response is shown in Fig. 6 and we assume that it represents an estimate of the true plant's gain response. From Fig.6, it is clear that 1 st elastic mode which exists around $1(\mathrm{~Hz})$ is dominant. In this case, we assume that the nominal model consists of the rigid mode and 1st elastic mode based on the
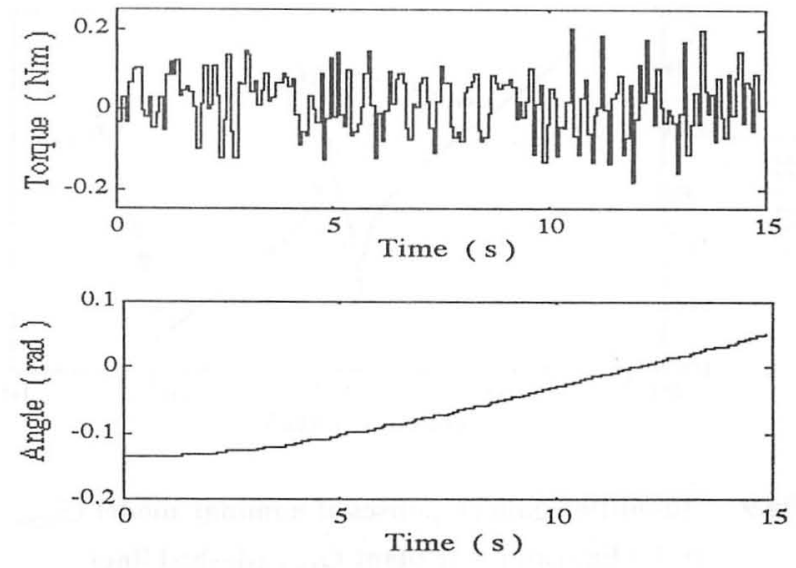

Fig.7 Dicimated input-output data for $d=4$

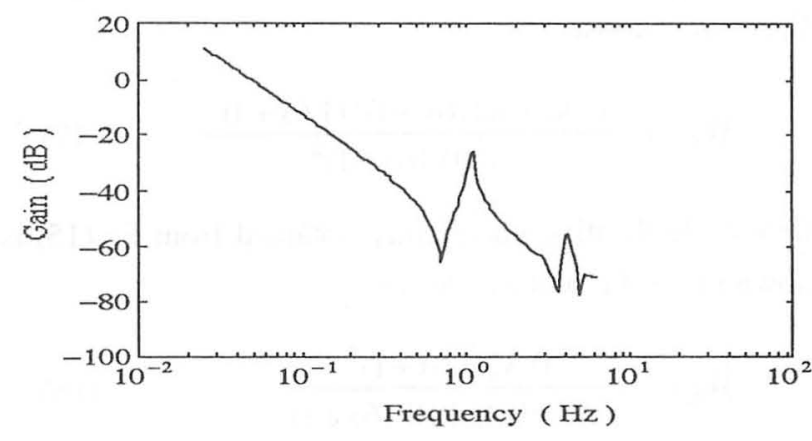

Fig.8 Identified gain response based on decimated data by using 10th order model

control requirement. Therefore, the nominal model transfer function is 4th order.

Step 2 : Fig. 7 shows the decimated input-output data for $d=4$, which corresponds to the case of $f_{s}=12.5(\mathrm{~Hz})$. So the sampling period $T$ is transformed to $T^{\prime}=4 T$ $=0.08(\mathrm{sec})$. Fig. 8 shows the identified gain response based on the decimated data, where 10th order model is used. From this results, modal parameters such that the inertia moment, the natural frequency, and so on, are calculated, so as to obtain the 4 th order continuous-time nominal model. By converting the continuous-time model to the discrete-time model with sampling period $T$, the following model is obtained.

$$
\hat{G}_{\text {nom }}(q)=\frac{10^{-4}\left(0.44 q^{-1}-0.44 q^{-2}-0.44 q^{-3}+0.44 q^{-4}\right)}{\left(1-q^{-1}\right)^{2}\left(1-1.98 q^{-1}+0.99 q^{-2}\right)}
$$

Gain response calculated from Eq. (16) is shown in Fig.9, where $\hat{G}_{\text {true }}$ is also shown which is obtained in Step 1.

Step 3 : Additive uncertainty calculated by Eq. (14) is 


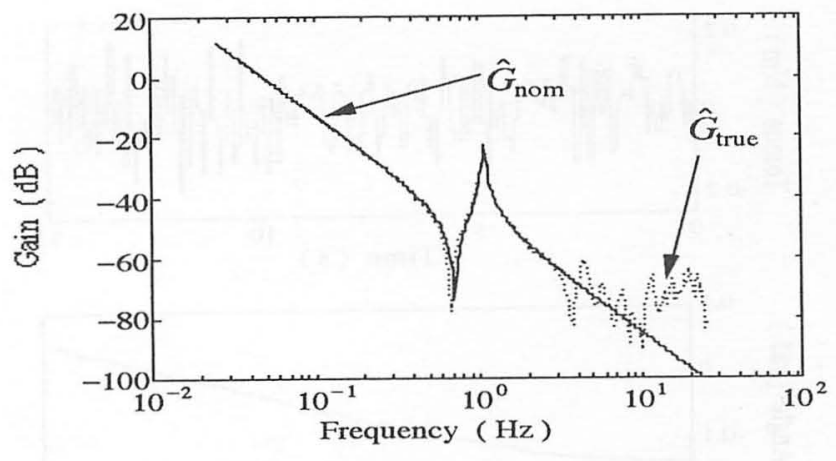

Fig.9 Identified gain responses of nominal model $\hat{G}_{\text {nom }}$ (solid line) and true plant $\hat{G}_{\text {true }}$ (dashed line)

shown in Fig.10. As an frequency weighting function $W_{A}(s)$, we choose

$$
W_{A}(s)=\frac{0.063(0.016 s+1)^{2}(1.6 s+1)^{2}}{s^{2}(0.16 s+1)^{2}}
$$

Also, multiplicative uncertainty obtained from Eq.(15) is shown in Fig.11, and we choose

$$
W_{M}(s)=\frac{0.32(1.6 s+1)^{2}}{(0.080 s+1)(0.16 s+1)}
$$

The identified frequency weighting functions are considered to be reasonable from a robust control design point of view.

\section{CONCLUSIONS}

In this paper, the simple robust identification method for the LSS based on the least-squares method in cooperation with the decimation has been proposed. The main advantage of the proposed method is that it needs no a priori in-

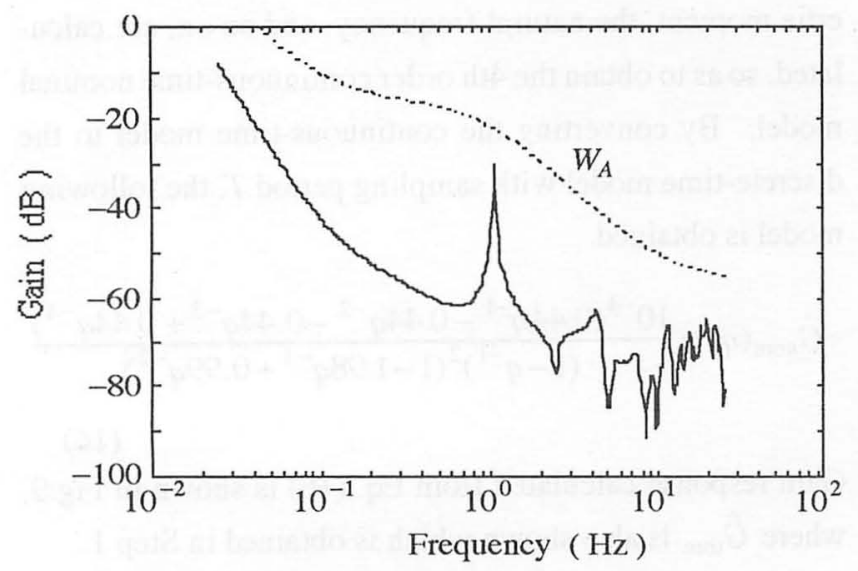

Fig.10 Identified additive uncertainty formation on the model uncertainty. Therefore, the method is considered to be applied to the practical problems easily.

In this paper, the effectiveness of the method is examined through the ground-based test model. Moreover, the on-orbit identification experiments including the evaluation for the model uncertainty using the ETS-VI are planned. Therefore, the effectiveness will be examined through the real spacecraft in the near future.

\section{References}

[1] P.Dorato and R.K.Yedavalli ed. : Recent Advances in Robust Control, IEEE Press (1990)

[2] Special Issue on System Identification for Robust Control, IEEE Trans. on Automat. Contr., AC-37-7 (1992)

[3] N.L.Gupta, et al. : Modeling, control and system identification methods for flexible structures, AGARD-AG-260, 12 1-41 (1981)

[4] S.Adachi,et al. : System identification by multi-decimation (in Japanese), Trans. of SICE, 26, 9, 1029/1035 (1990)

[5] L.Ljung : System Identification - Theory for the Users, Prentice-Hall (1987)

[6] S.Adachi,et al. : Identification and control experiments for a large space structure, 11th IFAC World Congress, 5, 22/27, Tallin, USSR (1990)

[7] S.M.Joshi : Control of Large Flexible Space Structures. Lecture Notes in Control and Information Sciences, SpringerVerlag (1989)

[8] S.Adachi and A.Sano : Least squares estimation of impulse response in consideration on ill-condition of input autocovariance matrix (in Japanese), Trans. of SICE, 22, $11,1156 / 1161(1986)$

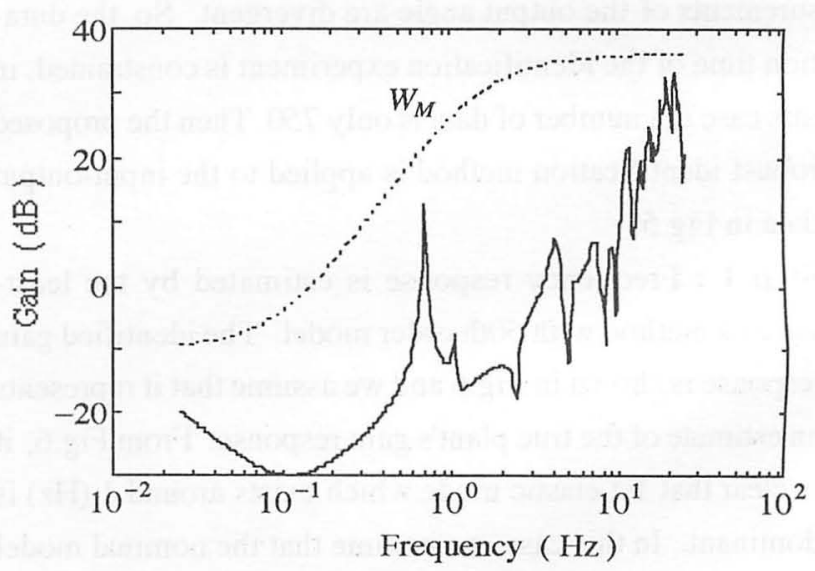

Fig.11 Identified multiplicative uncertainty 